\title{
Impact of repeated dose of stable iodine in an in utero rat model using a metabolomic approach
}

\author{
Clément Rosique $^{1}$, Dalila Lebsir $^{2}$, Maâmar Souidi ${ }^{2}$, and Jean-Charles Martin ${ }^{1}$ \\ ${ }^{1}$ Aix Marseille Univ, INSERM, INRA, C2VN, Marseille, France \\ ${ }^{2}$ Institut de Radioprotection et de Sûreté Nucléaire (IRSN), PRP-HOM, SRBE, LRTOX, 92260 \\ Fontenay-aux-Roses, France
}

\begin{abstract}
The Fukushima nuclear power plant blast resulted in the release of ${ }^{131}$ Iodine for several weeks. This unexpected issue challenged the iodin doctrine [1], in which the countermeasure is to provide a unique iodine tablet to saturate thyroid during the radioactive contamination not expected to last more than several hours. A new doctrine must be implemented to take into account such case of extended exposure based on repeated iodine administration with adapted dosage. But repeated administration of iodine can block the thyroid [2] and few scientific evidences regarding repeated iodine administration (and its potential undesirable effect) are at our disposal [3]. Moreover, unborn and young children are at high risk during a nuclear incident: it is currently recognized that one of the risks of exposure to radioactive iodine is the development of thyroid cancer, especially when exposure occurred during childhood [4]. Their protection is a main priority. Our goal was to evaluate the potential undesirable effects of such repeated iodine administration in the offspring using an untargeted metabolomic approach on a rat reproductive model.

Pregnant rats received repeated doses of potassium iodine (KI group: $1 \mathrm{mg} / \mathrm{kg} / 24 \mathrm{~h}$ ) or water for injection (control group) for 8 days. The potential metabolic disruption was investigated in the offspring 30 days after weaning.

Using LC-MS, we compared the blood's metabolite composition between KI and control male rats; using a high throughput annotation procedure with an in-house databank, 264 metabolites were annotated (based on retention time and exact mass), combined in 52 functional biological modules/pathways, and converted into corresponding scores using a PLS multiblock algorithm (see Fig. 1).
\end{abstract}




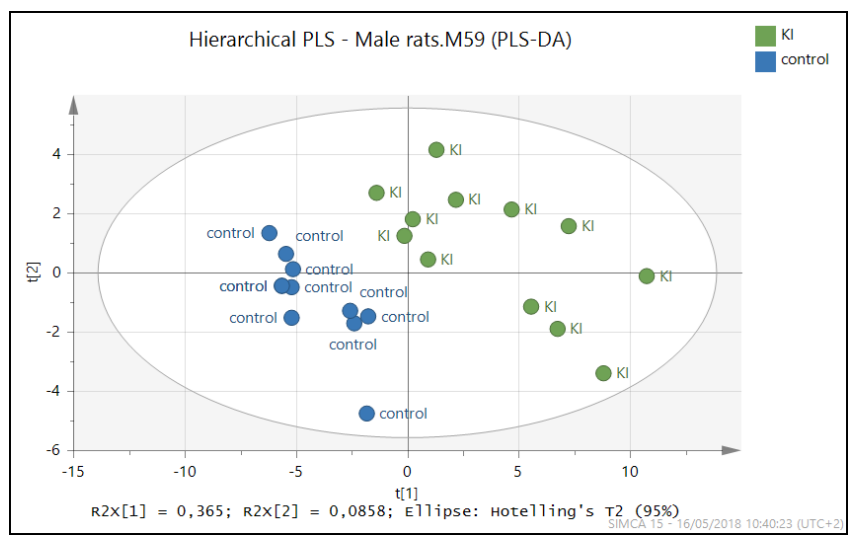

Fig. 1. Hierarchical PLS-DA based on 51 functional biological modules, CV-ANOVA p-value $=$ 0.0018

Running a random forests test, we found 19 modules significantly impacted by the KI treatment (VIP values $>0.05$ ) including pathways of redox status, aminoacids, TCA cycle or oxidative stress. These findings indicated a prenatal effect of KI administration that lasted over the long term (adolescence). Whether or not these outcomes are pathological is unknown.

\section{References}

[1] World Health Organization, « The World Health Report 1999 » (1999).

[2] S. Dreger, M. Pfinder, L. Christianson, S. K. Lhachimi, et H. Zeeb, « The effects of iodine blocking following nuclear accidents on thyroid cancer, hypothyroidism, and benign thyroid nodules: design of a systematic review », Systematic Reviews, vol. 4, $\mathrm{n}^{\circ}$ 1 (2015).

[3] P. Mitrou, S. A. Raptis, et G. Dimitriadis, « Thyroid disease in older people », Maturitas, vol. 70, $\mathrm{n}^{\mathrm{O}}$ 1, p. 5- 9 (2011).

[4] E. K. Cahoon et al., " Risk of Thyroid Nodules in Residents of Belarus Exposed to Chernobyl Fallout as Children and Adolescents », The Journal of Clinical Endocrinology \& Metabolism, vol. 102, n 7, p. 2207 - 2217 (2017). 